\title{
Una nueva especie de Leucheria (Asteraceae), endémica de Chile
}

\author{
LILIANA KATINAS ${ }^{1}$, JORGE V. CRISCI ${ }^{1}$ y ALICIA MARTICORENA²
}

\begin{abstract}
Summary: A new species of Leucheria (Asteraceae), endemic to Chile. A new species of Leucheria Lag. (Asteraceae, Nassauvieae), L. meladensis Katinas, Crisci \& A.E. Martic., with entire, elliptic, petiolate, scarcely pubescent leaves is described and illustrated. This would be the only species endemic to Reserva Nacional Bellotos del Melado, at $35^{\circ} \mathrm{S}$, in Linares province of the Maule region, Chile. A key to the species of Leucheria that inhabit the Linares province is presented.
\end{abstract}

Key words: Compositae, Chile, endemism, Nassauvieae.

Resumen: Se describe e ilustra una nueva especie de Leucheria Lag. (Asteraceae, Nassauvieae), L. meladensis Katinas, Crisci \& A.E. Martic., con hojas enteras, elípticas, pecioladas y levemente pubescentes. Esta sería la única especie endémica de la Reserva Nacional Bellotos del Melado, a los $35^{\circ} \mathrm{S}$, en la provincia de Linares, región del Maule en Chile. Se presenta una clave de las especies que habitan la provincia de Linares.

Palabras clave: Compositae, Chile, endemismo, Nassauvieae.

\section{INTRODUCCIÓN}

El género Leucheria Lag. (Asteraceae, Nassauvieae) se compone de 47 especies distribuidas en las regiones andino-patagónicas desde el Perú hasta el sur de Chile y Argentina, incluyendo algunas islas subantárticas (Crisci, 1976). De estas especies, 25 son compartidas entre Argentina y Chile y 17 son exclusivamente chilenas (Moreira-Muñoz et al., 2012).

Leucheria se caracteriza por su hábito herbáceo, con hojas dispuestas en una roseta basal, corolas bilabiadas, anteras sagitadas, estilos truncados con una coronita apical de pelos colectores y el papus plumoso (Katinas et al., 2008a). Además, la mayoría de las especies de Leucheria presenta una característica única en la tribu Nassauvieae que es la curiosa disposición de las filarias más internas del involucro como si fueran páleas del receptáculo (Crisci, 1976; Katinas et al., 2008b). Estas filarias

1División Plantas Vasculares, Museo de La Plata, Paseo del Bosque s/n, 1900 La Plata, Argentina. E-mail: katinas@fcnym.unlp.edu.ar

${ }^{2}$ Herbario CONC, Departamento de Botánica, Universidad de Concepción, Casilla 160-C, Concepción, Chile varían su orientación sobre el receptáculo rodeando a las flores del margen, ya sea con la cara cóncava hacia el centro del capítulo, con la cara cóncava hacia el exterior del capítulo, o dispuestas en forma perpendicular al centro del capítulo.

El género fue revisado por Crisci (1976) pero, dado que se halla en preparación una actualización de la taxonomía de Leucheria, se están analizando además ejemplares colectados luego de la revisión de 1976. Durante el análisis de material de Leucheria depositado en el herbario del Departamento de Botánica de la Universidad de Concepción, Chile (CONC), se halló un ejemplar con una morfología foliar que se aleja de la del resto de las especies. Dado que los caracteres reproductivos de corolas, estilos y anteras son muy homogéneos en el género, las características de las hojas son importantes para diferenciar a las especies. En este caso, las hojas enteras, elípticas y pecioladas son diferentes a las de las otras especies de Leucheria. Por otro lado, sus flores bilabiadas, el estilo característico de los miembros de Nassauvieae (Fig. 1A), el papus plumoso (Fig. 1B), y algunas filarias internas del involucro con la cara cóncava mirando al exterior del capítulo no dejan lugar a dudas que pertenece al género Leucheria. 

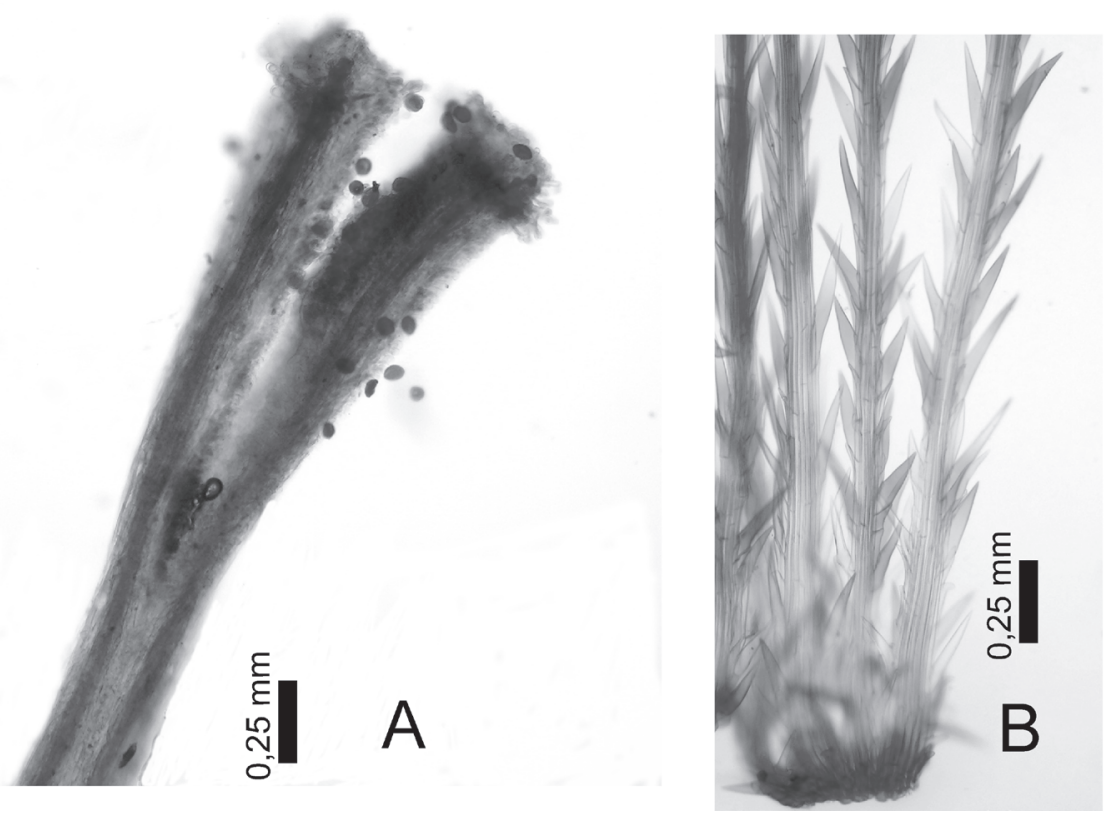

Fig. 1. A, B. Leucheria meladensis, fotografías al microscopio óptico. A, porción superior del estilo, típico de la tribu Nassauvieae, con dos ramas truncadas con una coronita de pelos colectores de polen en el ápice. $B$, porción inferior del papus mostrando las cerdas plumosas, unidas en su base en un anillo ( $A, B$, Arroyo et al. 994988, CONC).

Este ejemplar presenta además otro rasgo interesante. Fue colectado en el año 1999 por Mary Kalin Arroyo y colaboradores ( $n^{\circ}$ 994988, CONC) en la Reserva Nacional Bellotos del Melado ubicada a los $35^{\circ} \mathrm{S}$ en la provincia de Linares (región del Maule) de Chile. El nombre de la reserva se debe a que posee una de las pocas poblaciones del "belloto del sur" (Beilschmiedia berteroana (Gay) Kosterm., Lauraceae), un árbol endémico de Chile bajo la categoría En Peligro (Hechenleitner et al., 2005) y monumento natural desde 1995 (Muñoz et al., 1996). El nombre "Melado" hace referencia al río Melado de color amarillento que nace en la cordillera de los Andes, corre por la provincia de Linares y desagua en el río Ancoa, y al cerro El Melado con una altura de $2910 \mathrm{~m}$ ubicado en el límite norte de la Reserva (cf. Fig. 1 de Arroyo et al., 2000).

El ejemplar había sido determinado como $L$. hieracioides, otra especie de Leucheria que habita en la Reserva (Arroyo et al., 2000), pero con su estado de nueva especie pasaría a constituir el único taxón exclusivamente endémico de la Reserva, y a estar bajo la protección de la misma.

\section{Material y Métodos}

Este estudio se basa en el material analizado del herbario CONC (Holmgren et al., 1990), de donde se tomaron datos de localidad, fecha, colector y ecología del ambiente. Los órganos vegetativos y reproductivos fueron hervidos previos a su medición. Flores, frutos y papus se aclararon en hipoclorito de sodio diluido y se colorearon con safranina al 2\%. El montaje de los materiales se realizó en glicerina diluida al $10 \%$. Las observaciones y los dibujos de las partes morfológicas y anatómicas se hicieron con un microscopio estereoscópico Nikon SMZ 1000 y con un microscopio óptico Nikon Eclipse E200, ambos equipados con cámara clara. Las fotografías se tomaron con una cámara Nikon Coolpix S10.

\section{Resultados}

A continuación se describe la nueva especie y se presenta una clave de las especies de Leucheria que habitan la provincia de Linares, en Chile. 


\section{Katinas et al. -Nueva Leucheria (Asteraceae) de Chile}

Leucheria meladensis Katinas, Crisci \& A.E. Martic., nov. sp. (Fig. 2)

Tipo: Chile, Región del Maule, Provincia de Linares, Reserva Nacional Bellotos del Melado, $35^{\circ}$ 51' S, 71º 06' W, 19-XII-1999, M. T. K. Arroyo, P. MacPherson, M. Mihoc, A. Humaña \& C. Valdivia 994988 (Holotypus CONC 149089!).

Herba perennis, erecta, rhizomate obliquo, radicante. Folia radicalia petiolata, lamina integra, elpitica vel oblanceolata, 18-22 mm longa x 7-13 mm lata, glabra. Capitulis longe pedunculatis, ad apices ramorum solitariis, pedunculi dichotome diviso. Receptaculum margine paleatum, paleis subellipticus, involucri foliola interiora simulantibus. Involucrum 2-3-seriatum. Corollis bilabiatis. Stigmata bipartita, ramis semiteretibus, apice dilatato-truncatis, penicillatis. Pappo plumoso, ima basi concretae in annulum.

Hierba perenne, de ca. $20 \mathrm{~cm}$ alt., rizomatosa, caulescente con tallos ramificados o bifurcados en la parte superior. Hojas con venación pinnada, lámina simple, entera, elíptica o levemente obovada, margen entero, ápice redondeado, levemente acuminado, levemente estrigosas en el haz, araneosas en el envés; hojas inferiores dispuestas en roseta, generalmente rodeadas por restos de pecíolos de hojas viejas y secas, lámina de $18-22 \times 7-13 \mathrm{~mm}$, pecioladas, pecíolo de 25-35 mm long, envainador; hojas superiores alternas, sésiles, lámina de 10-30 x 4-10 mm, menores las más cercanas a los capítulos. Capítulos homógamos, discoides, en cimas 2-céfalas, laxas, largamente pedunculados. Involucro acampanado; filarias 2-3 seriadas, imbricadas, elípticas, glabras, estrigoso-pubescentes, las externas de 3-4 x 1-1,5 $\mathrm{mm}$, las internas de 6-7 mm x 1,5-2 mm, la tercera serie formada por algunas filarias que funcionan como páleas, con la cara convexa hacia el centro del capítulo y encerrando una o más flores con la segunda serie. Flores isomorfas, perfectas, corola blanca o amarillenta en el material herborizado, de 10-11 mm long, bilabiada, glabra, labio exterior 3-dentado, de 4-6 mm long, alargado en las flores marginales, labio interior bífido, de 2-2,5 mm long. Anteras sagitadas, de 1,8-2 $\mathrm{mm}$ long, apéndice conectival apical de 2-2,3 $\mathrm{mm}$ long, lanceolado, agudo, tecas terminadas inferiormente en largas colas, de 1-1,5 $\mathrm{mm}$ long, glabras, endotecio radial. Estilo de 6-7 mm long, bífido, ramas de 1-1,5 mm long, con papilas estigmáticas en la cara interna, truncadas y con una coronita de pelos colectores de polen en el ápice, con estilopodio globoso en la base del estilo. Cipselas de 2-4 mm long, elípticas u obovoides, superficie tuberculada por células epidérmicas prominentes, papiloso-pubescentes, con pelos gemelos, de 135-250 $\mu \mathrm{m}$, sin carpopodio basal. Papus blanco, formado por una serie de cerdas, de 5-6 mm long, plumosas, unidas en su base en un anillo.

El epíteto específico hace referencia al río Melado y al cerro Melado, que dan parte del nombre a la Reserva Nacional Bellotos del Melado, Chile, donde fue colectado el ejemplar tipo.

Especie endémica de la Reserva Nacional Bellotos del Melado de Chile, situada en la región del Maule, provincia de Linares, Comuna de Colbún (Fig. 3). Esta área se localiza en el extremo sur de la región con clima de tipo mediterráneo y con bosque de tipo caducifolio templado. Leucheria meladensis fue hallada en el sotobosque del bosque deciduo semiabierto (Arroyo et al., 2000) de Cryptocarya alba (Molina) Looser ("peumo", Lauraceae), Lomatia hirsuta (Lam.) Diels ("radal", Proteaceae) y Austrocedrus chilensis (D. Don) Pic. Serm. \& Bizarri ("ciprés de la cordillera", Cupressaceae), a los 1080 $\mathrm{m}$ de altura.

Hasta ahora sólo se conoce el ejemplar tipo de L. meladensis depositado en $\mathrm{CONC}$, de modo que futuros estudios de campo indicarán si ésta es una especie en peligro o no desde el punto de vista de su conservación de acuerdo al número de poblaciones existentes. Afortunadamente, de ser una especie vulnerable o en peligro, ya se encontraría protegida en una reserva nacional.

Leucheria meladensis comparte el hábito de hierba perenne y caulescente, y las hojas enteras con L. garciana, L. gilliesii, L. integrifolia y $L$. lithospermifolia, pero la morfología de las hojas es diferente. En L. meladensis el limbo es anchamente elíptico u obovado y no acompaña al pecíolo, mientras que en las otras especies el limbo es muy alargado, generalmente linear-elíptico o linearoblanceolado y acompaña al pecíolo hasta la base formando un angosto pseudopecíolo. Asimismo, en estas últimas especies las hojas suelen ser lanosas y comúnmente paucidentadas o paucilobuladas, hallándose ocasionalmente alguna hoja de margen entero, mientras que en L. meladensis las hojas son menos pubescentes y el limbo es entero. 
Bol. Soc. Argent. Bot. 53 (1) 2018

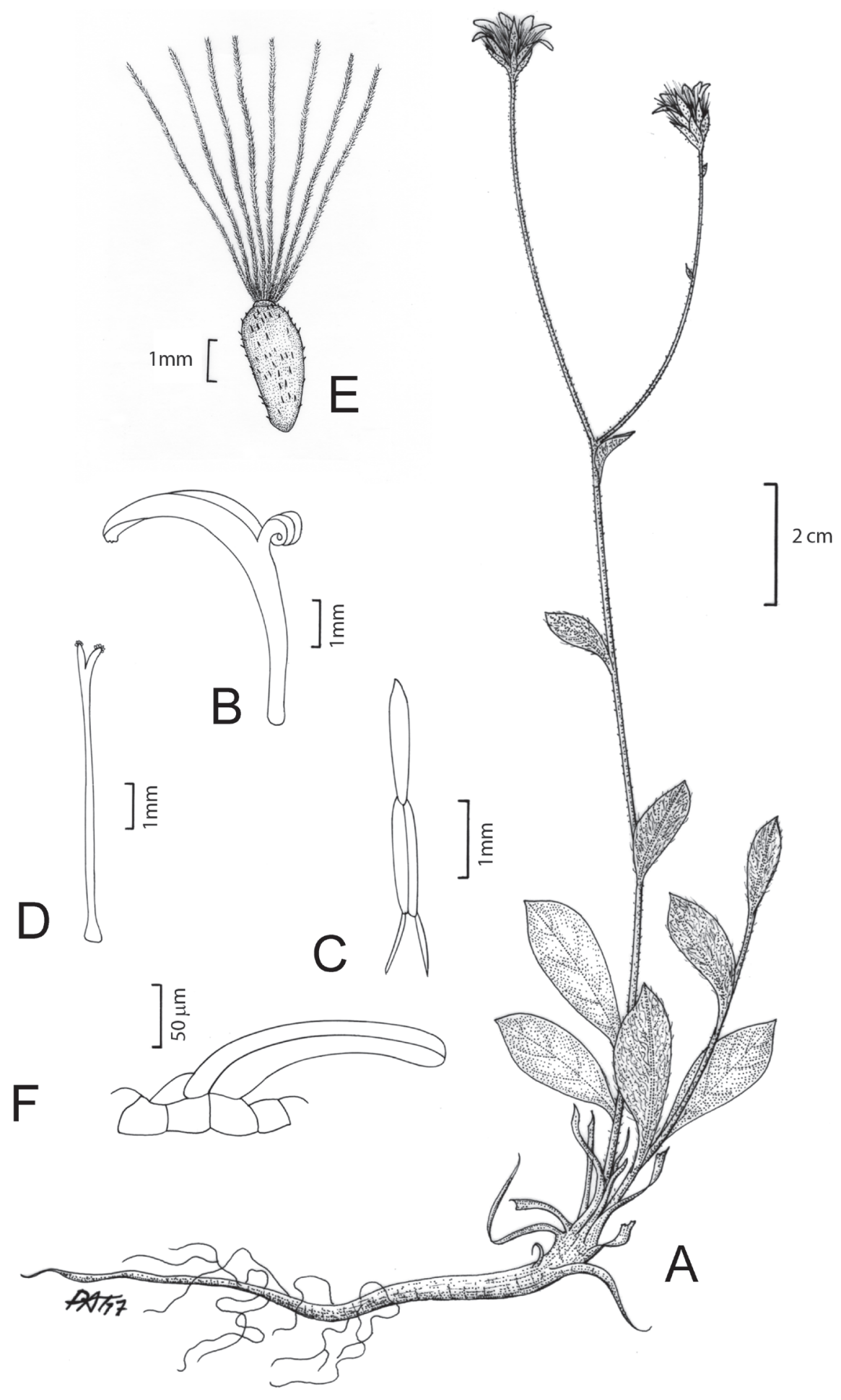

Fig. 2. Leucheria meladensis. A, hábito. B, corola. C, antera. D, estilo. E, fruto con papus. F, pelo gemelo del fruto (A-F, Arroyo et al. 994988, CONC). 


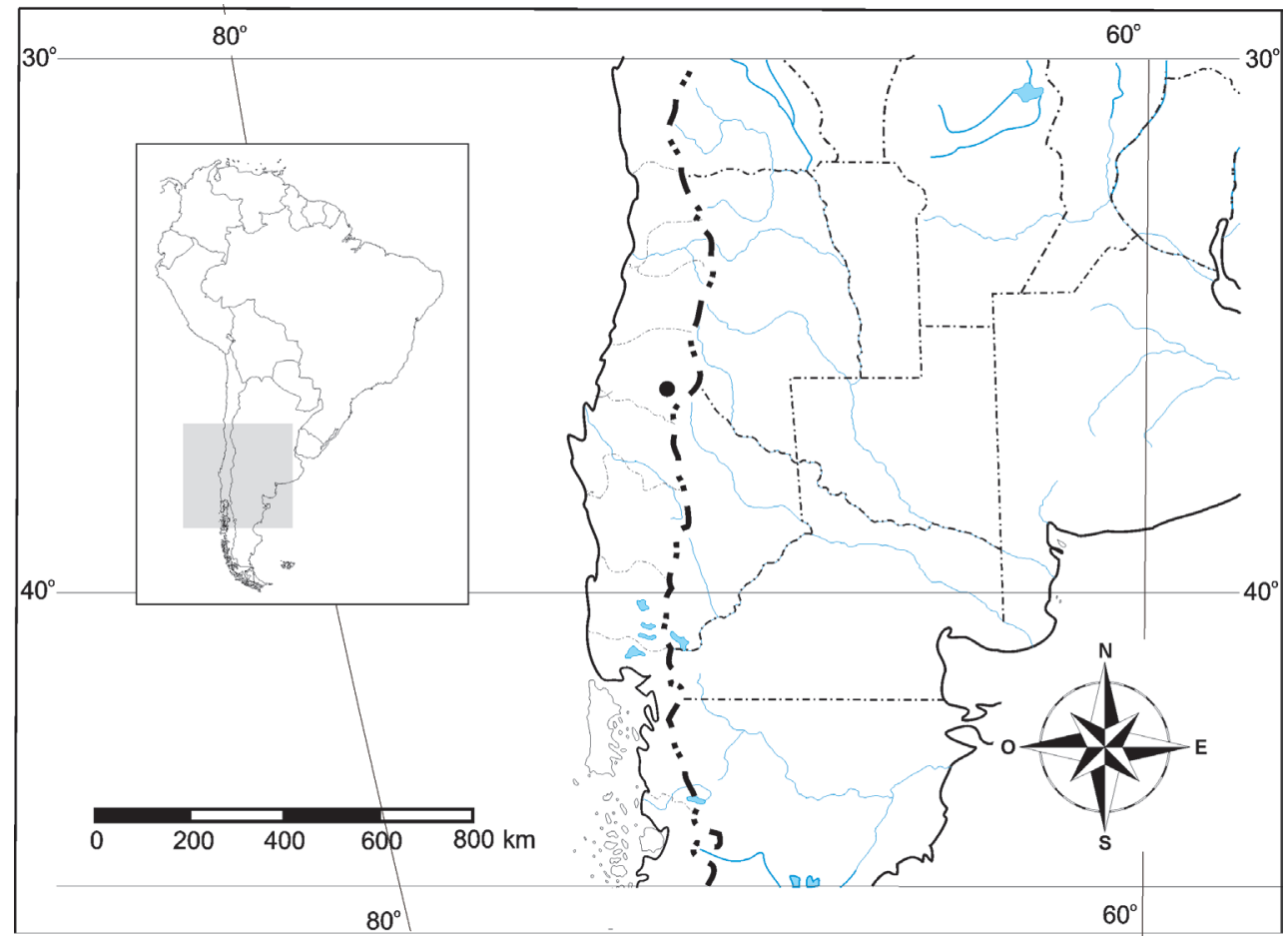

Fig. 3. Leucheria meladensis. Mapa de distribución.

\section{Clave de las especies de Leucheria de la provincia de Linares, Chile}

1. Hojas enteras a paucilobadas.

2. Hojas usualmente lanosas, más abundantemente en el envés, linear-oblanceoladas. Tallos y filarias del involucro lanosas.

3. Inflorescencias de hasta 15 capítulos. Capítulos de hasta $8 \mathrm{~mm}$ alt.

4. Hojas rígidas, pectinadas. Corolas rosadas a rojas.

L. viscida

4'. Hojas membranosas, enteras o algo dentadas. Corolas blancas a rosadas. .... L. lithospermifolia 3'. Inflorescencias de más de 15 capítulos. Capítulos de 12-15 mm alt. L. integrifolia

$2 '$. Hojas levemente estrigosas en el haz, araneosas en el envés, elípticas. Tallos y filarias del involucro no lanosas.

L. meladensis

1'. Hojas conspicuamente partidas.

5. Capítulos pequeños, de hasta 6(-7) $\mathrm{mm}$ alt. Hojas pinnatisectas.

L. amoena

5'. Capítulos más grandes, 7-20 mm alt. Hojas runcinadas.

6. Involucro de 7-10 $\mathrm{mm}$ de alt.

L. hieracioides

6 '. Involucro mayor de $10 \mathrm{~mm}$ alt.

L. glacialis 


\section{Agradecimientos}

Agradecemos a los editores y revisores por sus sugerencias. Este trabajo se realizó gracias al apoyo del Consejo Nacional de Investigaciones Científicas y Técnicas (CONICET), Agencia Nacional de Promoción Científica y Tecnológica (PICT 201201683), y Facultad de Ciencias Naturales y Museo, UNLP para LK y JVC.

\section{Bibliografía}

ARROYO, M. T. K., O. MATTHEI, C. MARTICORENA, M. MUÑOZ, F. PÉREZ \& A. M. HUMAÑA. 2000. La flora vascular de la Reserva Nacional Bellotos del Melado. VII Región, Chile: Un catálogo documentado. Gayana, Bot. 57: 117-139.

CRISCI, J. V. 1976. Revisión del género Leucheria (Compositae: Mutisieae). Darwiniana 20: 9-126.

HECHENLEITNER V. P., M. F. GARDNER, P. I. THOMAS, C. ECHEVERRÍA, B. ESCOBAR, P. BROWNLESS \& C. MARTÍNEZ A. 2005. Plantas amenazadas del centro-sur de Chile: Distribución, conservación y propagación. 1ra ed. Universidad Austral de Chile y Real Jardín Botánico de Edimburgo, Chile, 188 pp.
HOLMGREN, P. K., N. H. HOLMGREN \& L. C. BARNETT. 1990. Index Herbariorum, Part I: The herbaria of the world. 8th ed. New York Botanical Garden, Bronx, 693 pp.

KATINAS, L., J. PRUSKI, G. SANCHO \& M. C. TELLERÍA. 2008a. The subfamily Mutisioideae (Asteraceae). Bot. Rev. 74: 469-716.

KATINAS, L., J. V. CRISCI, R. SCHMIDT JABAILY, C. WILLIAMS, J. WALKER, B. DREW, J. M. BONIFACINO \& K. J. SYTSMA. 2008b. Evolution of secondary heads in Nassauviinae (Asteraceae, Mutisieae). Amer. J. Bot. 95: 229-240.

MOREIRA-MUÑOZ, A., V. MORALES \& M. MUÑOZSCHICK. 2012. Actualización sistemática y distribución geográfica de Mutisioideae (Asteraceae) de Chile. Gayana, Bot. 69: 9-29.

MUÑOZ, M., H. NÚÑEZ \& J. YÁÑEZ (eds.) 1996. Libro rojo de los sitios prioritarios para la conservación de la diversidad biológica en Chile. Ministerio de Agricultura, Corporación Nacional Forestal, Santiago, 203 pp.

Recibido el 2 de agosto de 2017, aceptado el 2 de octubre de 2017. Editor: Diego Gutierrez. 